\title{
ON SOME NONEXTENDABLE DERIVATIONS OF THE GAUGE-INVARIANT CAR ALGEBRA ${ }^{1}$
}

BY

GEOFFREY L. PRICE

\begin{abstract}
We provide examples of some approximately inner, commutative *-derivations which are generators on the gauge-invariant CAR algebra but which have no closed densely-defined extensions to the CAR. Necessary conditions are given for a class of generators on the GICAR algebra to extend to closed *-derivations on the CAR.
\end{abstract}

1. Introduction. Given a pair $\delta, \delta^{\prime}$ of closed $*$-derivations on a $C^{*}$-algebra $\mathscr{A}$, we say that $\delta^{\prime}$ is an extension of $\delta$ if its domain $D\left(\delta^{\prime}\right)$ contains $D(\delta)$ and its restriction $\left.\delta^{\prime}\right|_{D(\delta)}$ coincides with $\delta$. We say that $\delta$ is extendable if it admits an extension $\delta^{\prime}$ which is the infinitesimal generator of a strongly continuous one-parameter group of *-automorphisms. (For brevity, we refer to $\delta^{\prime}$ as a generator.) Recently a number of articles $[3,4,6-9,11]$ has appeared concerning the extendability of *-derivations commuting with a compact group $\left\{\alpha_{g}: g \in G\right\}$ of $*$-automorphisms of $\mathscr{A}$, i.e., $\alpha_{g}$ leaves $D(\delta)$ globally invariant and $\alpha_{g}(\delta x)=\delta\left(\alpha_{g} x\right)$, all $x \in D(\delta)$. A very recent result of Bratteli and Jørgensen [3] says that if $G$ is abelian and $\mathscr{A}^{G}$, the subalgebra of elements fixed by $G$, is $\mathrm{AF}$, then under the assumption that $\left.\delta\right|_{\mathscr{A}^{G}}$ is a generator, $\delta$ itself must also be a generator. Moreover, in certain more restrictive cases the authors show that any two generator extensions of $\left.\delta\right|_{\mathscr{A}^{G}}$ to $\mathscr{A}$ which commute with $G$ are related by a one-parameter subgroup of the action of $G$.

The latter result suggests the following problems. Let $\left(\mathscr{A}, G, \mathscr{A}^{G}\right)$ be as above, and suppose $\delta_{G}$ is a closed $*$-derivation on $\mathscr{A}^{G}$ which generates a $C^{*}$-dynamics. Then must it follow that there is a densely defined closed $*$-derivation on $\mathscr{A}$ which restricts to $\delta_{G}$ on $\mathscr{A}^{G}$ ? In particular, are there extensions commuting with the action of $G$ ?

In this paper we consider the particular situation where $\mathscr{A}$ is the CAR algebra (a UHF algebra of Glimm type $2^{\infty}$ ), $G=\mathrm{T}$, the circle group, and $\mathscr{A}^{\mathrm{T}}=\mathscr{A}^{0}$ is the GICAR algebra (gauge-invariant subalgebra of the canonical anticommutation relations algebra). We exhibit a class of commutative $*$-derivations on $\mathscr{A}^{0}$ (in the sense of [13]) which generate a $C^{*}$-dynamics on $\mathscr{A}^{0}$ but have no closed extensions to the CAR (Corollary 4.1).

Some of our techniques are inspired by the results in $[1,12,14]$, and we thank Robert T. Powers for acquainting us with the material therein. We also wish to

Received by the editors January $1,1983$.

1980 Mathematics Subject Classification. Primary 47B47.

Key words and phrases. Derivation, CAR algebra, GICAR algebra, generator.

${ }^{1}$ Supported in part by NSF Grant No. MCS-8202290. 
record our thanks to Fred Goodman, R. T. Powers and Palle Jørgensen for helpful conversations at the April 1982 CBMS conference in Iowa, and we are grateful to $O$. Bratteli for sending us a preprint of [3]. Finally, we thank S. Sakai for pointing out a serious error in the original manuscript.

2. Preliminaries and notation. We recall some facts needed from [1] and [2] about the GICAR algebra and introduce some notation. Let $B$ be the $2 \times 2$ matrix algebra over $\mathrm{C}$ with matrix units $\left\{e_{i j}: 1 \leqslant i, j \leqslant 2\right\}$ satisfying the identities (i) $e_{i j} e_{r s}=\delta_{j r} e_{i s}$, and (ii) $e_{11}+e_{22}=1$. For $k \in \mathbf{N}$, let $B_{k}$ be a copy of $B$ with corresponding matrix units $\left\{e_{i j}^{k}\right\}$. Denote the $2^{n} \times 2^{n}$ matrix algebra $\otimes_{k=1}^{n} B_{k}$ by $\mathfrak{A}_{n}$, with matrix units consisting of the tensors $e_{i_{1} j_{1}}^{1} \otimes \cdots \otimes e_{i_{n} j_{n}}^{n}$. Then we have the inclusions $\mathfrak{A}_{1} \subseteq \mathfrak{U}_{2} \subseteq$ $\cdots$ and the uniform closure of their union, $\mathfrak{A}=\otimes_{k \geqslant 1}^{*} B_{k}$, is a UHF algebra of Glimm type $2^{\infty}$, the CAR algebra (cf. [10]).

For $\theta \in \mathrm{T}$ let $u_{\theta} \in B$ be the unitary $\exp (i \theta / 2) e_{11}+\exp (-i \theta / 2) e_{22}$, and let $u_{\theta}^{k}$ be the corresponding elements of the $B_{k}$. Then the mapping $\theta \mapsto \alpha_{\theta}=\otimes_{k \geqslant 1} \operatorname{Ad}\left(u_{\theta}^{k}\right)$ is a strongly continuous representation of $T$ as product automorphisms on $\mathfrak{A}$ (the gauge group of automorphisms). A straightforward argument shows that

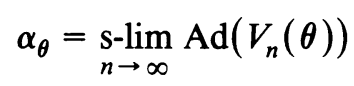

on $\mathfrak{A}$, where

$$
V_{n}(\theta)=\prod_{k=1}^{n}\left(e^{i \theta / 2} e_{11}^{k}+e^{-i \theta / 2} e_{22}^{k}\right)
$$

We denote by $\mathfrak{A}^{0}$ the AF $C^{*}$-subalgebra of elements fixed by the gauge group (called the GICAR algebra). $\mathfrak{U}^{0}=\bigcup_{n \geqslant 1} \mathfrak{U}_{n}^{0}$, where $\mathfrak{U}_{n}^{0}=\mathfrak{U}_{n} \cap \mathfrak{U}^{0} . \mathfrak{U}_{n}^{0}$ is generated by elements of the form $e_{i i}^{k}$ and $e_{i j}^{k} \otimes e_{j i}^{l}$, for $1 \leqslant i, j \leqslant 2,1 \leqslant k, l \leqslant n$ : in particular, a matrix unit $e_{i_{1} j_{1}}^{1} \otimes \cdots \otimes e_{i_{n} j_{n}}^{n}$ lies in $\mathfrak{A}_{n}^{0}$ if and only if $\sum_{r=1}^{n}\left(i_{r}-j_{r}\right)=0$.

For fixed $n \in \mathbf{N}, \mathfrak{U}_{n}^{0}$ decomposes as a direct sum $\mathfrak{U}_{n}^{0}=\sum_{k=0}^{n} M_{n, k}$ of $\left(\begin{array}{l}n \\ k\end{array}\right) \times\left(\begin{array}{l}n \\ k\end{array}\right)$ matrix algebras $M_{n, k}$ (where $\left(\begin{array}{l}n \\ k\end{array}\right)$ are the binomial coefficients). $M_{n, k}$ is spanned by matrix units $e_{i_{1} j_{1}}^{1} \otimes \cdots \otimes e_{i_{n} j_{n}}^{n} \in \mathfrak{U}_{n}^{0}$ where $k=\#\left\{r: 1 \leqslant r \leqslant n, i_{r}=1\right\}$. Let $E_{k}^{n}$ be the maximal projection in $M_{n, k}$ : then $E_{k}^{n}$ is the sum of the $\left(\begin{array}{l}n \\ k\end{array}\right)$ (diagonal) matrix units $M_{n, k}$ of the form $e_{i_{1} i_{1}}^{1} \otimes \cdots \otimes e_{i_{n} i_{n}}^{n}$. Clearly $\Sigma_{k=0}^{n} E_{k}^{n}=1$, and the $E_{k}^{n}$ generate the center $\mathscr{Z}\left(\mathfrak{U}_{n}^{0}\right)$ of $\mathfrak{U}_{n}^{0}$. An easy observation shows that the following identities hold for $n \in \mathbf{N}$ :

$$
\begin{aligned}
& E_{0}^{n+1}=E_{0}^{n} e_{22}^{n+1}, \\
& E_{k}^{n+1}=E_{k}^{n} e_{22}^{n+1}+E_{k-1}^{n} e_{11}^{n+1}, \quad 1 \leqslant k \leqslant n, \\
& E_{n+1}^{n+1}=E_{n}^{n} e_{11}^{n+1} .
\end{aligned}
$$

Given any set of operators $\mathfrak{B}$ (in $\mathfrak{A}$ or $\mathfrak{A}^{0}$ ), we define $\mathfrak{B}^{c}$ (respectively, $\mathfrak{B}^{c_{0}}$ ), the relative commutant of $\mathfrak{B}$ in $\mathfrak{A}$ (respectively, in $\mathfrak{A}^{0}$ ), by

$$
\mathfrak{B}^{c}=\{x \in \mathfrak{U}: x b=b x, \text { all } b \in \mathfrak{B}\}
$$

(respectively, $\mathfrak{B}^{c_{0}}=\left\{x \in \mathfrak{A}^{0}: x b=b x\right.$, all $\left.b \in \mathfrak{B}\right\}$ ).

Finally, we introduce the class of infinitesimal generators to be studied here. Let $\mathscr{S}$ be the set of all sequences $\left\{h_{n}: n \in \mathbf{N}\right\}$ of selfadjoint elements $h_{n} \in \mathfrak{A}_{n}^{0}$ such that for 
all $n \in \mathbf{N}, h_{n+1}-h_{n} \in\left(\mathfrak{U}_{n}^{0}\right)^{c_{0}}$. Then it follows easily that the sequence $\left(h_{n}\right)$ consists of a mutually commuting family of selfadjoint elements (note that $h_{m}-h_{n}$ $\left.\in\left(\mathfrak{U}_{n}^{0}\right)^{c_{0}}, m \geqslant n\right)$ and the same observation shows that for fixed $n \in \mathbf{N}$, and any $x_{n} \in \mathfrak{U}_{n}^{0}$,

$$
\begin{aligned}
e^{i t h_{m}}\left(x_{n}\right) e^{-i t h_{m}} & =e^{i t h_{n}} e^{i t\left(h_{m}-h_{n}\right)}\left(x_{n}\right) e^{-i t\left(h_{m}-h_{n}\right)} e^{-i t h_{n}} \\
& =e^{i t h_{n}}\left(x_{n}\right) e^{-i t h_{n}} \quad \text { for } m \geqslant n
\end{aligned}
$$

For arbitrary $x \in \mathfrak{U}^{0}$ and $\varepsilon>0$, choose $n$ sufficiently large so that $\left\|x-x_{n}\right\|<\varepsilon / 2$ for some $x_{n} \in \mathfrak{A}_{n}^{0}$. Then for $p \geqslant q \geqslant n$ we have, by (3),

$$
\begin{aligned}
& \left\|e^{i t h_{p}}(x) e^{-i t h_{p}}-e^{i t h_{q}}(x) e^{-i t h_{q}}\right\| \\
& \leqslant \\
& \leqslant e^{i t h_{p}}\left(x-x_{n}\right) e^{-i t h_{p}}\|+\| e^{i t h_{p}}\left(x_{n}\right) e^{-i t h_{p}}-e^{i t h_{q}}\left(x_{n}\right) e^{-i t h_{q}} \| \\
& \quad+\left\|e^{i t h_{q}}\left(x-x_{n}\right) e^{-i t h_{q}}\right\| \\
& <\varepsilon / 2+0+\varepsilon / 2=\varepsilon,
\end{aligned}
$$

so that the sequence $\left\{e^{i t h_{p}}(x) e^{-i t h_{p}}: p \in \mathbf{N}\right\}$ is Cauchy, all $x \in \mathfrak{A}^{0}$. Using this and other similar approximation arguments we have

LEMma 2.1. Let $\left(h_{n}\right) \in \mathscr{S}$ be a sequence defined as above. Then the limit

$$
\beta_{t}(x)=\lim _{n \rightarrow \infty} e^{i t h_{n}}(x) e^{-i t h_{n}}
$$

exists for all $t \in \mathbf{R}, x \in \mathfrak{A}^{0}$. The set $\left\{\beta_{t}: t \in \mathbf{R}\right\}$ is a strongly continuous group of *-automorphisms of $\mathfrak{A}^{0}$.

For a fixed sequence $h=\left(h_{n}\right) \in \mathscr{S}$, let $\left\{\beta_{t}^{h}: t \in \mathbf{R}\right\}$ be the corresponding one-parameter group of $*$-automorphisms of $\mathfrak{A}^{0}$, and $\delta_{h}$ the corresponding infinitesimal generator. In Theorem 4.1 (see also the Remark following Corollary 2.3) we shall show that some derivations $\delta_{k}$ fail to extend to generators on the CAR. Roughly speaking, the reason that $\delta_{h}$ may not lift is that, whereas the operators $\exp \left(i t\left[h_{n}-h_{m}\right]\right)$ tend to commute with finite tensors in $\mathfrak{U}^{0}$ for large $n, m$, the same may not hold for finite tensors in $\mathfrak{A}$. As a preliminary we examine the structure of the sequence $\left(h_{n}\right)$.

LEMMA 2.2. Let $\left(h_{n}\right) \in \mathscr{S}$. Then for all $n \in \mathbf{N}$ there exist constants $\lambda_{k}^{n}, \mu_{k}^{n} \in \mathbf{R}$ such that

$$
h_{n+1}-h_{n}=\sum_{k=0}^{n} E_{k}^{n}\left(\lambda_{k}^{n} e_{11}^{n+1}+\mu_{k}^{n} e_{22}^{n+1}\right) .
$$

Proof. Since $\left(h_{n+1}-h_{n}\right) \in \mathfrak{A}_{n+1}^{0}$ (hence in $\left.\mathfrak{A}_{n+1}\right)$, we may write $\left(h_{n+1}-h_{n}\right)=$ $\sum_{r, s=1}^{2} x_{r s} e_{r s}^{n+1}$, where $x_{r s} \in \mathfrak{U}_{n}, r, s=1$, 2. Moreover, $\left(h_{n+1}-h_{n}\right) \in\left(\mathfrak{U}_{n}^{0}\right)^{c_{0}}$ (hence in $\left.\left(\mathfrak{U}_{n}^{0}\right)^{c}\right)$ by hypothesis; but clearly $e_{r s}^{n+1} \in\left(\mathfrak{U}_{n}^{0}\right)^{c}$, so it follows directly that for each $r, s, x_{r s} \in\left(\mathfrak{U}_{n}^{0}\right)^{c} \cap \mathfrak{U}_{n}$. By [1, Lemma 3.7], $\left(\mathfrak{U}_{n}^{0}\right)^{c} \cap \mathfrak{U}_{n}=\dot{\mathscr{Z}}\left(\mathfrak{U}_{n}^{0}\right)$, so that each 
$x_{r s}$ is a linear combination of the $E_{k}^{n}, 0 \leqslant k \leqslant n$. In particular, $x_{r s} \in \mathfrak{A}^{0}$, but then $\alpha_{\theta}\left(x_{r s}\right)=x_{r s}$, all $\theta \in \mathrm{T}$, and therefore

$$
\begin{aligned}
h_{n+1}-h_{n} & =\frac{1}{2 \pi} \int_{0}^{2 \pi} \alpha_{\theta}\left(h_{n+1}-h_{n}\right) d \theta=\frac{1}{2 \pi} \sum_{r, s=1}^{2} x_{r s} \int_{0}^{2 \pi} \alpha_{\theta}\left(e_{r s}^{n+1}\right) d \theta \\
& =\frac{1}{2 \pi} \sum_{r, s=1}^{2} x_{r s} \int_{0}^{2 \pi} e^{-i(r-s) \theta} e_{r s}^{n+1} d \theta=x_{11} e_{11}^{n+1}+x_{22} e_{22}^{n+1} .
\end{aligned}
$$

Finally, the selfadjointness of $h_{n+1}-h_{n}$ implies the same for the $x_{j j}$, so the $x_{j j}$ are real linear combinations of the projections $E_{k}^{n}$, and the result now follows immediately.

LEMMA 2.3. Suppose $h=\left(h_{n}\right)$ and $h^{\prime}=\left(h_{n}^{\prime}\right)$ are two sequences in $\mathscr{S}$. Then the generators $\delta_{h}, \delta_{h^{\prime}}$, on $\mathfrak{U}^{0}$ coincide if and only if, for each $n \in \mathbf{N}, h_{n}-h_{n}^{\prime} \in \mathscr{Z}\left(\mathfrak{U}_{n}^{0}\right)$.

PRoof. If $\delta_{h}=\delta_{h^{\prime}}$, then $\beta_{t}^{h}$ and $\beta_{t}^{h^{\prime}}$ coincide on $\mathfrak{U}_{n}^{0}$, all $t \in \mathbf{R}, n \in \mathbf{N}$. Let $x \in \mathfrak{A}_{n}^{0}$. Then, by (3),

$$
e^{i t h_{n}}(x) e^{-i t h_{n}}=e^{i t h_{n}^{\prime}}(x) e^{-i t h_{n}^{\prime}} \quad \text { or } \quad e^{-i t h_{n}^{\prime}}\left(e^{i t h_{n}}\right) \in\left(\mathfrak{U}_{n}^{0}\right)^{c_{0}}, \quad \text { all } t \in \mathbf{R} .
$$

Taking $\left.(d / d t)\right|_{t=0}$ then gives $h_{n}-h_{n}^{\prime} \in\left(\mathfrak{A}_{n}^{0}\right)^{c_{0}}$. On the other hand, if $h_{n}-h_{n}^{\prime} \in$ $\mathscr{Z}\left(\mathfrak{U}_{n}^{0}\right)$, then clearly $\operatorname{Ad}\left(e^{i t h_{n}}\right)=\operatorname{Ad}\left(e^{i t h_{n}^{\prime}}\right)$ on $\mathfrak{U}_{n}^{0}$. Hence $\beta_{t}^{h^{\prime}}$ agrees with $\beta_{t}^{h}$ on $\mathfrak{U}_{n}^{0}$, all $n \in \mathbf{N}$, and, by continuity, $\beta_{t}^{h}=\beta_{t}^{h^{\prime}}$, hence $\delta_{h}=\delta_{h^{\prime}}$.

CoROllaRY. Given a sequence $h^{\prime}=\left(h_{n}^{\prime}\right) \in \mathscr{S}$ with generator $\delta_{h^{\prime}}$, there exists a unique sequence $h=\left(h_{n}\right) \in \mathscr{S}$ with $\delta_{h}=\delta_{h^{\prime}}$ such that

$$
\begin{gathered}
h_{1}=0, \\
h_{k+1}-h_{k}=\sum_{l=0}^{k} E_{l}^{k} \gamma_{l}^{k} e_{11}^{k+1}, \quad k \in \mathbf{N},
\end{gathered}
$$

where $\gamma_{l}^{k} \in \mathbf{R}, 0 \leqslant l \leqslant k$, and $\gamma_{k}^{k}=\gamma_{k-1}^{k}$.

Proof. We construct $\left(h_{n}\right)$ inductively. Since $\mathfrak{A}_{1}^{0}=\mathscr{Z}\left(\mathfrak{A}_{1}^{0}\right)$, we may apply the lemma to assume $h_{1}=0$. Suppose $\left\{h_{k}: 1 \leqslant k \leqslant n\right\}$ have been chosen to satisfy (i), (ii), and also $h_{k}-h_{k}^{\prime} \in \mathscr{Z}\left(\mathfrak{A}_{k}^{0}\right)$. Using (4) and the assumption $h_{n}-h_{n}^{\prime} \in \mathscr{Z}\left(\mathfrak{U}_{n}^{0}\right)$, we may assume there are $\xi_{l}^{n}, \eta_{l}^{n} \in \mathbf{R}(0 \leqslant l \leqslant n)$ such that

$$
h_{n+1}^{\prime}-h_{n}=\left(h_{n+1}^{\prime}-h_{n}^{\prime}\right)+\left(h_{n}^{\prime}-h_{n}\right)=\sum_{l=0}^{n} E_{l}^{n}\left(\xi_{l}^{n} e_{11}^{n+1}+\eta_{l}^{n} e_{22}^{n+1}\right) \text {. }
$$

Choose

$$
h_{n+1}=h_{n+1}^{\prime}-\left[\sum_{l=0}^{n} \eta_{l}^{n} E_{l}^{n+1}\right]+\left(\xi_{n-1}^{n}-\xi_{n}^{n}-\eta_{n}^{n}\right) E_{n+1}^{n+1}
$$

(note that $h_{n+1}-h_{n+1}^{\prime} \in \mathscr{Z}\left(\mathfrak{A}_{n+1}^{0}\right)$ ). Then using (2) a straightforward calculation shows $h_{n+1}-h_{n}$ satisfies (ii) of the corollary, where $\gamma_{l}^{n}=\xi_{l}^{n}-\eta_{l+1}^{n}$, for $0 \leqslant l \leqslant$ $n-1$, and $\gamma_{n}^{n}=\xi_{n-1}^{n}-\eta_{n}^{n}=\gamma_{n-1}^{n}$. Hence the induction step holds.

To prove uniqueness suppose $h, h^{\prime \prime}$ satisfy properties (i) and (ii) above and let $n>1$ be the first index such that $h_{n} \neq h_{n}^{\prime \prime}$. Then note that $h_{n}-h_{n}^{\prime \prime} \notin\left(\mathfrak{A}_{n}^{0}\right)^{c_{0}}$, so $\delta_{h} \neq \delta_{h^{\prime \prime}}$ by the lemma. 
REMARK. Let $[\mathscr{S}$ ] denote the set of equivalence classes of sequences in $\mathscr{S}$ which generate the same dynamics on $\mathfrak{U}^{0}$. Let $\mathscr{S}_{0}$ be the subset of $\mathscr{S}$ consisting of those sequences $s$ satisfying properties (i) and (ii) of the corollary. Then by the lemma and its corollary there exists for each $\diamond \in[\mathscr{S}]$ a unique sequence $h \in \mathscr{S}_{0}$ such that $[h]=\diamond$.

Suppose $h=\left(h_{n}\right) \in \mathscr{S}_{0}, \delta_{h}$ is the associated derivation on $\mathfrak{A}^{0}$, and $\left\{\beta_{t}^{h}: t \in \mathbf{R}\right\}$ is the one-parameter group of automorphisms of $\mathfrak{U}^{0}$ generated by $\delta_{h}$. It follows easily from Corollary 2.3 that for each $n \in \mathbf{N}, h_{n}$ lies in the maximal abelian subalgebra of $\mathfrak{A}_{n}$ generated by the diagonal elements $e_{i i}^{k}, 1 \leqslant k \leqslant n, i=1,2$. Hence, for $t \in \mathbf{R}$,

$$
\beta_{t}^{h}\left(e_{i i}^{k}\right)=\lim _{n} \exp \left(i t h_{n}\right) e_{i i}^{k} \exp \left(-i t h_{n}\right)=\lim _{n} e_{i i}^{k}=e_{i i}^{k},
$$

so that $\beta_{t}^{h}$ fixes the m.a.s.a. of $\mathfrak{A}^{0}$ generated by the diagonal elements. Now suppose that $\left(\lambda_{n}\right)$ is a sequence of real numbers, with $\lambda_{1}=0$, and $h^{\prime}=\left(h_{n}^{\prime}\right)$ is a new sequence of operators defined by setting $h_{n}^{\prime}=h_{n}+\sum_{k=1}^{n} \lambda_{k} e_{11}^{k}$. Observe that $\left(h^{\prime}\right) \in$ $\mathscr{S}_{0}$-note

$$
h_{n+1}^{\prime}-h_{n}^{\prime}=h_{n+1}-h_{n}+\lambda_{n+1} e_{11}^{n+1}
$$

and

$$
\lambda_{n+1} e_{11}^{n+1}=\sum_{l=0}^{n} E_{l}^{n} \lambda_{n+1} e_{11}^{n+1} .
$$

We show that the generator $\delta_{h^{\prime}}$ on $\mathfrak{A}^{0}$ has an extension to a generator on $\mathfrak{A}$ if and only if the same holds for $\delta_{h}$. For suppose $\delta_{h}$ has a generator extension to $\mathfrak{A}$, and let $\left\{\hat{\beta}_{t}: t \in \mathbf{R}\right\}$ be the corresponding one-parameter group on $\mathfrak{A}$. Then it is clear that $\hat{\beta}_{\left.t\right|_{\mathfrak{x}}}=\beta_{t}^{h}$, so that, in particular, $\hat{\beta}_{t}\left(e_{i i}^{k}\right)=e_{i i}^{k}, k \in \mathbf{N}$. Define another group $\left\{\rho_{t}\right.$ : $t \in \mathbf{R}\}$ of automorphisms on $\mathfrak{A}$ by setting, for $x \in \mathfrak{A}$,

$$
\rho_{t}(x)=\lim _{n} \exp \left(\text { it } \sum_{k=1}^{n} \lambda_{k} e_{11}^{k}\right) x \cdot \exp \left(-i t \sum_{k=1}^{n} \lambda_{k} e_{11}^{k}\right) \text {. }
$$

It is straightforward to show that this limit converges, and $\left\{\rho_{t}\right\}$ is a group of product automorphisms on $\mathfrak{A}$, where

$$
\rho_{t}\left(x_{k}\right)=\operatorname{Ad}\left[\exp \left(i t \lambda_{k} e_{11}^{k}\right)\right]\left(x_{k}\right), \quad x_{k} \in B_{k} .
$$

Furthermore, we have

$$
\begin{aligned}
\hat{\beta}_{s}\left(\rho_{t}(x)\right) & =\lim _{n} \hat{\beta}_{s}\left[\exp \left(i t \sum_{k=1}^{n} \lambda_{k} e_{11}^{k}\right) \cdot x \cdot \exp \left(-i t \sum_{k=1}^{n} \lambda_{k} e_{11}^{k}\right)\right] \\
& =\lim _{n} \beta_{s}^{h}\left[\exp \left(i t \sum_{k=1}^{n} \lambda_{k} e_{11}^{k}\right)\right] \cdot \hat{\beta}_{s}(x) \cdot \beta_{s}^{h}\left[\exp \left(-i t \sum_{k=1}^{n} \lambda_{k} e_{11}^{k}\right)\right] \\
& =\lim _{n} \exp \left(i t \sum_{k=1}^{n} \lambda_{k} e_{11}^{k}\right) \cdot \hat{\beta}_{s}(x) \cdot \exp \left(-i t \sum_{k=1}^{n} \lambda_{k} e_{11}^{k}\right) \\
& =\rho_{t}\left(\hat{\beta}_{s}(x)\right),
\end{aligned}
$$


so $\rho_{t} \circ \hat{\beta}_{s}=\hat{\beta}_{s} \circ \rho_{t}, s, t \in \mathbf{R}$, from which it follows that the set $\left\{\hat{\beta}_{t}^{\prime}=\rho_{t} \circ \hat{\beta}_{t}: t \in \mathbf{R}\right\}$ is itself a one-parameter group of automorphisms of $\mathfrak{A}$. Finally, for $x \in \mathfrak{A}_{n}^{0}$, we note that

$$
\begin{aligned}
\lim _{t \rightarrow 0} \frac{\rho_{t}\left(\hat{\beta}_{t}(x)\right)-x}{t} & =\lim _{t \rightarrow 0} \frac{\rho_{t}\left(\beta_{t}^{h}(x)\right)-\beta_{t}^{h}(x)}{t}+\frac{\beta_{t}^{h}(x)-x}{t} \\
& =\lim _{t \rightarrow 0}\left(\beta_{t}^{h} \frac{\left[\rho_{t}(x)-x\right]}{t}\right)+\delta_{h}(x) \\
& =\operatorname{Ad}\left[i \sum_{k=1}^{n} \lambda_{k} e_{11}^{k}, x\right]+\delta_{h}(x)=\delta_{h^{\prime}}(x) .
\end{aligned}
$$

Hence $\left\{\hat{\beta}_{t}^{\prime}\right\}$ is a one-parameter group on $\mathfrak{A}$ having a generator which extends $\delta_{h^{\prime}}$. The argument in the reverse direction is identical. Thus we have proved

LEMMA 2.4. Let $h, h^{\prime} \in \mathscr{S}_{0}$ be two sequences such that $h_{n}^{\prime}=h_{n}+\sum_{k=1}^{n} \lambda_{k} e_{11}^{k}$ for some sequence $\left(\lambda_{n}\right)$ of real numbers. Then $\delta_{h}$ on $\mathfrak{U}^{0}$ has a generator extension to $\mathfrak{A}$ if and only if $\delta_{h^{\prime}}$ has one also.

Definition 2.1. Denote by $\mathscr{S}_{00}$ the subset of $\mathscr{S}_{0}$ consisting of all $h^{\prime}=\left(h_{n}^{\prime}\right)$ satisfying the conditions

(i') $h_{1}^{\prime}=0$, and

(ii') $h_{k+1}^{\prime}-h_{k}^{\prime}=\sum_{l=0}^{k} E_{l}^{k} \xi_{l}^{k} e_{11}^{k+1}, k \in \mathbf{N}$, where $\xi_{l}^{k} \in \mathbf{R}$, and $\xi_{k-1}^{k}=\xi_{k}^{k}=0$.

Given $h=\left(h_{n}\right) \in \mathscr{S}_{0}$, where $h_{k+1}-h_{k}=\sum_{l=0}^{k} E_{l}^{k} \gamma_{l}^{k} e_{11}^{k+1}$, as in (ii) of Corollary 2.3 , let $\left(\lambda_{n}\right)$ be the sequence of real numbers $\lambda_{n}=-\gamma_{n-1}^{n-1}$, and let $h_{n}^{\prime}=h_{n}+$ $\sum_{k=1}^{n} \lambda_{k} e_{11}^{k}$. Then one verifies easily that $h^{\prime}=\left(h_{n}^{\prime}\right)$ lies in $\mathscr{S}_{00}$ : indeed,

$$
h_{n+1}^{\prime}-h_{n}^{\prime}=\sum_{l=0}^{n} E_{l}^{n} \xi_{l}^{n} e_{11}^{n+1},
$$

where $\xi_{l}^{n}=\gamma_{l}^{n}-\gamma_{n}^{n}, 0 \leqslant l \leqslant n$, so that $h^{\prime}$ satisfies (i') and (ii') above. Using the preceding lemma and some technical results below, we shall be able in Theorem 4.1 and its corollary to determine a necessary condition for a generator $\delta_{h}\left(h \in \mathscr{S}_{0}\right)$ to extend to a closed $*$-derivation on $\mathfrak{A}$.

For the remainder of the section, fix $h=\left(h_{n}\right) \in \mathscr{S}_{00}$-i.e., $h_{1}=0$, and

$$
h_{k+1}-h_{k}=\sum_{l=0}^{k} E_{l}^{k} \xi_{l}^{k} e_{11}^{k+1}, \quad \xi_{k-1}^{k}=\xi_{k}^{k}=0,
$$

and let $\delta_{h}$ be the corresponding $*$-derivation generating the group $\left\{\beta_{t}=\beta_{t}^{h}: t \in \mathbf{R}\right\}$ on $\mathfrak{U}^{0}$. The following condition on the coefficients $\xi_{l}^{k}$ (which we shall call Condition C) ensures that $\delta_{h}$ extends to a generator on $\mathfrak{A}$ (Lemma 2.5).

Condition C. For $\delta>0$ there exists $N(=N(\delta) \in \mathbf{N})$ such that if

(a) $\left\{l_{j}: 1 \leqslant j \leqslant q\right\}$ is a strictly increasing (possibly infinite) set of integers with $l_{1} \geqslant N$, and if

(b) $r$ is any fixed integer, $0 \leqslant r<l_{1}$, then

$$
\left|\sum_{j=1}^{q}\left(\xi_{r+j}^{l_{j}}-\xi_{r+j-1}^{l_{j}}\right)\right|<\delta .
$$


Condition $\mathrm{C}$ clearly implies the following condition.

Condition $\mathrm{C}^{\prime}$. For $\delta>0$ there exists $N(=N(\delta) \in \mathbf{N})$ such that if

(a) $\left\{l_{j}: 1 \leqslant j \leqslant q\right\}$ is a strictly increasing finite set of integers with $l_{1} \geqslant N$, and

(b) $r$ is any fixed integer, $0 \leqslant r: l_{1}$, and

(c) $m$ is a fix 1 positive integer greater than $l_{q}+1$, then

$$
\left|\sum_{j=1}^{q}\left(\xi_{r+j}^{l_{j}}-\xi_{r+j-1}^{l_{j}}\right)-\left(\xi_{r+j}^{m+j-1}-\xi_{r+j-1}^{m+j-1}\right)\right|<\delta .
$$

Lemma 2.5. Let $h \in \mathscr{S}_{00}$ and $\beta_{t}, t \in \mathbf{R}$, be as above. Then if Condition $\mathrm{C}$ is satisfied, the generator $\delta_{h}$ on $\mathfrak{U}^{0}$ extends to a generator on $\mathfrak{A}$.

Proof. We begin by showing that the sequence $\left\{\exp \left(i t h_{k}\right)\left(e_{12}^{1}\right) \exp \left(-i t h_{k}\right)\right\}$ is uniformly convergent. For $\delta>0$, let $m>n>N(\delta)$. Then

(6) $\left\|\exp \left(i t h_{m+1}\right)\left(e_{12}^{1}\right) \exp \left(-i t h_{m+1}\right)-\exp \left(i t h_{n}\right)\left(e_{12}^{1}\right) \exp \left(-i t h_{n}\right)\right\|$

$$
=\left\|\exp \left(i t\left[h_{m+1}-h_{n}\right]\right)\left(e_{12}^{1}\right) \exp \left(-i t\left[h_{m+1}-h_{n}\right]\right)-e_{12}^{1}\right\| .
$$

Using (i') and (ii') of Definition 2.1, one has

$$
h_{m+1}-h_{n}=\sum_{k=n}^{m} \sum_{l=0}^{k} E_{l}^{k} \xi_{l}^{k} e_{11}^{k+1},
$$

so

$$
\begin{aligned}
\exp \left(i t\left[h_{m+1}-h_{n}\right]\right) & =\exp \left(i t \sum_{k=n}^{m} \sum_{l=0}^{k} E_{l}^{k} \xi_{l}^{k} e_{11}^{k+1}\right) \\
& =\prod_{k=n}^{m} \prod_{l=0}^{k} \exp \left(i t E_{l}^{k} \xi_{l}^{k} e_{11}^{k+1}\right) .
\end{aligned}
$$

Next note that $e_{12}^{1}$ may be decomposed as the sum

$$
\sum_{I \in \mathscr{I}} e_{12}^{1} e_{i_{2} i_{2}}^{2} \cdots e_{i_{m+1} i_{m+1}}^{m+1}=\sum_{I \in \mathscr{I}} e_{12}^{1} e_{I I},
$$

where $\mathscr{I}$ is the set of all $m$-tuples $I=\left(i_{2}, i_{3}, \ldots, i_{m+1}\right)$, with $i_{j}$ either 1 or 2 , and $e_{I I}=e_{i_{2} i_{2}}^{2} \cdots e_{i_{m+1} i_{m+1}}^{m+1}$. Fix one of these $I$, and suppose that

$$
r=\#\left\{j: 2 \leqslant j \leqslant n \text { and } i_{j}=1\right\} \text {. }
$$

Furthermore, let $\left\{l_{j}: 1 \leqslant j \leqslant q\right\}$ be the ordered set of indices $l, n \leqslant l \leqslant m$, such that $i_{l+1}=1$. Then using (2) one has, for $t \in \mathbf{R}, k \geqslant n$,

$$
\exp \left(i t E_{l}^{k} \xi_{l}^{k} e_{11}^{k+1}\right) e_{12}^{1} e_{I I}=\exp \left(i t \xi_{l}^{k}\right) e_{12}^{1} e_{I I},
$$

if both $i_{k+1}=1$ and $l=\left[1+\#\left\{j: 2 \leqslant j \leqslant k, i_{j}=1\right\}\right]$ hold, and

$$
\exp \left(i t E_{l}^{k} \xi_{l}^{k} e_{11}^{k+1}\right) e_{12}^{1} e_{I I}=e_{12}^{1} e_{I I},
$$

otherwise. Applying (7), we then have, by the preceding calculation,

$$
\begin{aligned}
\exp \left(i t\left[h_{m+1}-h_{n}\right]\right) e_{12}^{1} e_{I I} & =\exp \left(i t \xi_{r+1}^{l_{1}}\right) \exp \left(i t \xi_{r+2}^{l_{2}}\right) \cdots \exp \left(i t \xi_{r+q}^{l_{q}}\right) e_{12}^{1} e_{I I} \\
& =\exp \left(i t \sum_{j=1}^{q} \xi_{r+j}^{l_{j}}\right) e_{12}^{1} e_{I I} .
\end{aligned}
$$


A similar calculation gives

$$
e_{12}^{1} e_{I I} \exp \left(-i t\left[h_{m+1}-h_{n}\right]\right)=e_{12}^{1} e_{I I} \exp \left(-i t \sum_{j=1}^{q} \xi_{r+j-1}^{l_{j}}\right) .
$$

Combining these last two results, we then have

$$
\begin{aligned}
\exp \left(i t\left[h_{m+1}-h_{n}\right]\right) & \cdot e_{12}^{1} e_{I I} \exp \left(-i t\left[h_{m+1}-h_{n}\right]\right) \\
& =\exp \left(i t \sum_{j=1}^{q}\left(\xi_{r+j}^{l_{j}}-\xi_{r+j-1}^{l_{j}}\right)\right) \cdot e_{12}^{1} e_{I I} .
\end{aligned}
$$

Denote the coefficient on $e_{12}^{1} e_{I I}$ by $c_{I}(t)$. Then

$$
\begin{aligned}
\| \exp ( & \text { it } \left.\left[h_{m+1}-h_{n}\right]\right) e_{12}^{1} \exp \left(-i t\left[h_{m+1}-h_{n}\right]\right)-e_{12}^{1} \| \\
= & \left\|\sum_{I \in \mathscr{I}}\left[\exp \left(i t\left[h_{m+1}-h_{n}\right]\right) \cdot e_{12}^{1} e_{I I} \cdot \exp \left(-i t\left[h_{m+1}-h_{n}\right]\right)-e_{12}^{1} e_{I I}\right]\right\| \\
= & \left\|\sum_{I \in \mathscr{I}}\left(c_{I}(t)-1\right) e_{12}^{1} e_{I I}\right\| \leqslant\left\|e_{12}^{1}\right\|\left\|\sum_{I \in \mathscr{I}}\left(c_{I}(t)-1\right) e_{I I}\right\| \\
= & \max _{I \in \mathscr{I}}\left\{\left|c_{I}(t)-1\right|\right\},
\end{aligned}
$$

where the last equality follows from elementary spectral theory, using the fact that the $e_{I I}$ are mutually orthogonal projections. Using the condition of the lemma one sees that for fixed $t$, sufficiently large $n$, and all $I \in \mathscr{I}, c_{I}(t)$ can be made arbitrarily close to 1 , so that $\operatorname{Ad}\left(\exp \left(i t\left[h_{m+1}-h_{n}\right]\right)\right)\left(e_{12}^{1}\right)$ converges uniformly to $e_{12}^{1}$, for $n, m$ large. From (6) it now follows immediately that $\left\{\operatorname{Ad}\left(\exp \left(i t h_{n}\right)\right)\left(e_{12}^{1}\right)\right\}$ is a uniformly convergent sequence.

A similar argument shows that for any $k \in \mathbf{N}$, the sequence $\left\{\operatorname{Ad}\left(\exp \left(i t h_{n}\right)\right)\left(e_{12}^{k}\right)\right.$ : $n \in \mathbf{N}\}$ converges uniformly: taking adjoints, the same holds for $e_{21}^{k}$. In addition,

$$
\operatorname{Ad}\left(\exp \left(i t h_{n}\right)\right)\left(e_{i i}^{k}\right)=e_{i i}^{k}
$$

since $h_{n}$ and $e_{i i}^{k}$ lie in the m.a.s.a. of $\mathfrak{A}$ generated by the diagonal elements. Hence, $\left\{\operatorname{Ad}\left(\exp \left(i t h_{n}\right)\right)\left(e_{i j}^{k}\right)\right\}$ is uniformly convergent for all matrix units $e_{i j}^{k}$. One then easily extends these results to polynomials in the matrix units $e_{i j}^{k}$, whence a straightforward approximation argument shows that $\left\{\exp \left(i t h_{n}\right) \cdot x \cdot \exp \left(-i t h_{n}\right)\right\}$ is norm convergent for all $x \in \mathfrak{A}, t \in \mathbf{R}$. Therefore, the automorphism group $\left\{\beta_{t}: t \in \mathbf{R}\right\}$ extends to $\mathfrak{A}$, so that $\delta_{h}$ has a generator extension to $\mathfrak{U}$ as asserted.

3. A necessary condition for extendability. In this section we show that Condition $\mathrm{C}^{\prime}$ is necessary for a generator $\delta_{h}$ on $\mathfrak{U}^{0}\left(h \in \mathscr{S}_{00}\right)$ to have a generator extension to $\mathfrak{U}$. The following lemma is the main tool used to obtain this result. First we introduce some notation required for the lemma.

For indices $l$ and $p, 0 \leqslant l \leqslant p$, let $E p$ be the projection in $\mathfrak{A}_{p}^{0}$ defined as in the previous section. For $1 \leqslant k \leqslant p$ and $0 \leqslant l \leqslant p-1$, we define $F_{l}^{k, p}$ to be the projection in $\mathfrak{U}_{p}^{0}$ consisting of the sum of all diagonal elements in $\mathfrak{A}_{p}$ of the form

$$
e_{i_{1} i_{1}}^{1} \cdots e_{i_{k-1} i_{k-1}}^{k-1} e_{i_{k+1} i_{k+1}}^{k+1} \cdots e_{i_{p} i_{p}}^{p}
$$


where $l$ of the subscripts $i_{1}, \ldots, i_{k-1}, i_{k+1}, \ldots, i_{p}$ are equal to 1 . Then one observes that the following identities hold (see also (2)):

$$
\begin{aligned}
& E_{\delta}^{p}=e_{22}^{k} F_{0}^{k, p}, \\
& E^{p}=e_{11}^{k} F_{l-1}^{k, p}+e_{22}^{k} F_{l}^{k, p}, \quad 1 \leqslant l \leqslant p-1, \\
& E_{p}^{p}=e_{11}^{k} F_{p-1}^{k, p} .
\end{aligned}
$$

Note that $F_{l}^{k, p}$ commutes with all matrix units $e_{i j}^{k} \in B_{k}$.

LEMMA 3.1. Let $h=\left(h_{k}\right)$ be a sequence in $\mathscr{S}_{00}$, i.e., $h_{1}=0$, and

$$
h_{k+1}-h_{k}=\sum_{l=0}^{k} E_{l}^{k} \xi_{l}^{k} e_{11}^{k+1}, \quad \xi_{l}^{k} \in \mathbf{R}, \quad \text { and } \quad \xi_{k-1}^{k}=\xi_{k}^{k}=0 .
$$

Let $\beta_{t}=\beta_{t}^{h}, t \in \mathbf{R}$, be the group of automorphisms on $\mathfrak{A}^{0}$ generated by $\delta_{h}$.

\section{Then}

(i) $\beta_{t}\left(e_{i i}^{k}\right)=e_{i i}^{k}, k \in \mathbf{N}$, and

(ii) for positive integers $m>n+1$,

$$
\begin{aligned}
\beta_{l}\left(e_{21}^{n+1} e_{12}^{m+1}\right)= & \exp \left(-i t \sum_{l=0}^{n-1} E_{l}^{n} \xi_{l}^{n}\right) \cdot \exp \left(i t \sum_{j=n+1}^{m-1} \sum_{l=0}^{j-1} F_{l}^{n+1, j}\left(\xi_{l}^{j}-\xi_{l+1}^{j}\right) e_{11}^{j+1}\right) \\
& \cdot \exp \left(i t \sum_{l=0}^{m-1} F_{l}^{n+1, m} \xi_{l}^{m}\right) \cdot\left(e_{21}^{n+1} \cdot e_{12}^{m+1}\right) .
\end{aligned}
$$

Proof. Part (i) has already been obtained in (5).

To prove (ii) we begin by noting that since $e_{21}^{n+1} e_{12}^{m+1} \in \mathfrak{A}_{m+1}^{0}$, a calculation similar to (3) gives

$$
\begin{aligned}
\beta_{t}\left(e_{21}^{n+1} e_{12}^{m+1}\right) & =\exp \left(i t h_{m+1}\right)\left(e_{21}^{n+1} e_{12}^{m+1}\right) \exp \left(-i t h_{m+1}\right) \\
& =\exp \left(i t h_{m+1}\right)\left(e_{21}^{n+1} e_{12}^{m+1}\right) \exp \left(-i t\left[h_{m+1}-h_{m}\right]\right) \exp \left(-i t h_{m}\right) .
\end{aligned}
$$

Moreover,

$$
\begin{aligned}
\exp \left(-i t\left[h_{m+1}-h_{m}\right]\right) & =\exp \left(-i t \sum_{l=0}^{m} E_{l}^{m} \xi_{l}^{m} e_{11}^{m+1}\right) \\
& =\prod_{l=0}^{m} \exp \left(-i t E_{l}^{m} \xi_{l}^{m} e_{11}^{m+1}\right) \\
& =\prod_{l=0}^{m}\left(\left[I-E_{l}^{m} e_{11}^{m+1}\right]+\exp \left(-i t \xi_{l}^{m}\right) \cdot E_{l}^{m} e_{11}^{m+1}\right),
\end{aligned}
$$

so

$$
e_{12}^{m+1} \exp \left(-i t\left[h_{m+1}-h_{m}\right]\right)=e_{12}^{m+1}
$$

and therefore,

$$
\begin{aligned}
& \beta_{t}\left(e_{21}^{n+1} e_{12}^{m+1}\right)=\exp \left(i t h_{m+1}\right) \cdot e_{21}^{n+1} e_{12}^{m+1} \cdot \exp \left(-i t h_{m}\right) \\
& =\exp \left(i t h_{m+1}\right) \cdot e_{21}^{n+1} \cdot \exp \left(-i t h_{m}\right) \cdot e_{12}^{m+1} \\
& =\exp \left(i t\left[h_{m+1}-h_{n}\right]\right) \exp \left(i t h_{n}\right) e_{21}^{n+1} \exp \left(-i t h_{n}\right) \exp \left(-i t\left[h_{m}-h_{n}\right]\right) e_{12}^{m+1} \\
& \quad=\exp \left(i t\left[h_{m+1}-h_{n}\right]\right) e_{21}^{n+1} \exp \left(-i t\left[h_{m}-h_{n}\right]\right) e_{12}^{m+1} .
\end{aligned}
$$


(Note $\exp \left(-i t h_{r}\right) \in \mathfrak{A}_{r}$ and thus commutes with $e_{i j}^{r+1}, r \in \mathbf{N}$.) Therefore,

$$
\begin{aligned}
\beta_{l}\left(e_{21}^{n+1} e_{12}^{m+1}\right)= & \exp \left(i t\left[h_{m+1}-h_{n}\right]\right) e_{21}^{n+1} \exp \left(-i t\left[h_{m}-h_{n}\right]\right) e_{12}^{m+1} \\
= & \exp \left(i t \sum_{j=n}^{m} \sum_{l=0}^{j} E_{l}^{j} \xi_{l}^{j} e_{11}^{j+1}\right) e_{21}^{n+1} \exp \left(-i t \sum_{j=n}^{m-1} \sum_{l=0}^{j} E_{l}^{j} \xi_{l}^{j} e_{11}^{j+1}\right) \cdot e_{12}^{m+1} \\
= & \exp \left(i t \sum_{j=n+1}^{m} \sum_{l=0}^{j} E_{l}^{j} \xi_{l}^{j} e_{11}^{j+1}\right) e_{21}^{n+1} \exp \left(-i t \sum_{l=0}^{n} E_{l}^{n} \xi_{l}^{n} e_{11}^{n+1}\right) \\
& \cdot \exp \left(-i t \sum_{j=n+1}^{m-1} \sum_{l=0}^{j} E_{l}^{j} \xi_{l}^{j} e_{11}^{j+1}\right) \cdot e_{12}^{m+1} \\
= & \exp \left(-i t \sum_{l=0}^{n} E_{l}^{n} \xi_{l}^{n}\right) \exp \left(i t \sum_{j=n+1}^{m} \sum_{l=0}^{j} E_{l}^{j} \xi_{l}^{j} e_{11}^{j+1}\right) e_{21}^{n+1} \\
& \cdot \exp \left(-i t \sum_{j=n+1}^{m-1} \sum_{l=0}^{j} E_{l}^{j} \xi_{l}^{j} e_{11}^{j+1}\right) \cdot e_{12}^{m+1} \\
= & \exp \left(-i t \sum_{l=0}^{n} E_{l}^{n} \xi_{l}^{n}\right) \exp \left(i t \sum_{j=n}^{m} \sum_{l=0}^{j} E_{l}^{j} \xi_{l}^{j} e_{11}^{j+1}\right) e_{21}^{n+1} \\
& \cdot \exp \left(-i t \sum_{j=n+1}^{m-1} \sum_{l=1}^{j} E_{l}^{j} \xi_{l}^{j} e_{11}^{j+1}\right) \cdot e_{12}^{m+1} .
\end{aligned}
$$

Using (9) (and recalling $\xi_{j-1}^{j}=\xi_{j}^{j}=0$, all $j$ ) a straightforward calculation gives

$$
\begin{aligned}
& \exp \left(\text { it } \sum_{j=n+1}^{m-1} \sum_{l=0}^{j} E_{l} \xi_{l}^{j} e_{11}^{j+1}\right) e_{21}^{n+1} \exp \left(-i t \sum_{j=n+1}^{m-1} \sum_{l=1}^{j} E_{l}^{j} \xi_{l}^{j} e_{11}^{j+1}\right) \\
& \quad=\exp \left(i t \sum_{j=n+1}^{m-1} \sum_{l=0}^{j-1} F_{l}^{n+1, j} \xi_{l}^{j} e_{11}^{j+1}\right) e_{21}^{n+1} \exp \left(-i t \sum_{j=n+1}^{m-1} \sum_{l=1}^{j-1} F_{l-1}^{n+1, j} \xi_{l}^{j} e_{11}^{j+1}\right) \\
& =\exp \left(i t \sum_{j=n+1}^{m-1} \sum_{l=0}^{j-1} F_{l}^{n+1, j}\left(\xi_{l}^{j}-\xi_{l+1}^{j}\right) e_{11}^{j+1}\right) \cdot e_{21}^{n+1},
\end{aligned}
$$

and therefore,

$$
\begin{aligned}
\beta_{t}\left(e_{21}^{n+1} e_{12}^{m+1}\right)= & \exp \left(-i t \sum_{l=0}^{n} E_{l}^{n} \xi_{l}^{n}\right) \exp \left(i t \sum_{j=n+1}^{m-1} \sum_{l=0}^{j-1} F_{l}^{n+1, j}\left(\xi_{l}^{j}-\xi_{l+1}^{j}\right) e_{11}^{j+1}\right) \\
& \cdot \exp \left(i t \sum_{l=0}^{m} E_{l}^{m} \xi_{l}^{m} e_{11}^{m+1}\right) e_{21}^{n+1} e_{12}^{m+1}
\end{aligned}
$$


Finally, one has

$$
\begin{aligned}
\exp \left(i t \sum_{l=0}^{m} E_{l}^{m} \xi_{l}^{m} e_{11}^{m+1}\right) e_{21}^{n+1} e_{12}^{m+1} & =\exp \left(\text { it } \sum_{l=0}^{m} E_{l}^{m} \xi_{l}^{m}\right) e_{21}^{n+1} e_{12}^{m+1} \\
& =\exp \left(i t \sum_{l=0}^{m-1} F_{l}^{n+1, m} \xi_{l}^{m}\right) e_{21}^{n+1} e_{12}^{m+1}
\end{aligned}
$$

from (9). Combining this with the previous equation gives the desired form for $\beta_{t}\left(e_{21}^{n+1} e_{12}^{m+1}\right)$. This completes the lemma.

Suppose $\delta$ is a generator on $\mathfrak{A}$ with one-parameter group $\left\{\hat{\beta}_{t}: t \in \mathbf{R}\right\}$ extending $\delta_{h}$. Then it is straightforward to verify that $\hat{\beta}_{t_{\left.\right|_{\mathfrak{Y}} 0}}=\beta_{t}^{h}$. We consider $\hat{\beta}_{t}\left(e_{12}^{1}\right)$. For fixed $n$ we may write (see [5, Lemma 2])

$$
\hat{\beta}_{t}\left(e_{12}^{1}\right)=\sum_{I, J \in \mathscr{I}} e_{i_{1} j_{1}}^{1} \cdots e_{i_{n} j_{n}}^{n}\left(x_{I J}(t)\right)
$$

where $\mathscr{I}_{\text {is }}$ the set of $n$-tuples $I=\left(i_{1}, \ldots, i_{n}\right)$ with $i_{r} \in\{1,2\}$, and $x_{I J}(t) \in\left(\mathfrak{A}_{n}\right)^{c}$. By Lemma 3.1(i),

$$
\left(e_{11}^{1}\right) \hat{\beta}_{t}\left(e_{12}^{1}\right)\left(e_{22}^{1}\right)=\hat{\beta}_{t}\left(e_{11}^{1}\right) \hat{\beta}_{t}\left(e_{12}^{1}\right) \hat{\beta}_{t}\left(e_{22}^{1}\right)=\hat{\beta}_{t}\left(e_{11}^{1} e_{12}^{1} e_{22}^{1}\right)=\hat{\beta}_{t}\left(e_{12}^{1}\right),
$$

so we may assume that $i_{1}=1$ and $j_{1}=2$. Similarly, $\hat{\beta}_{t}\left(e_{12}^{1}\right)$ commutes with the projections $e_{k_{2} k_{2}}^{2} \cdots e_{k_{n} k_{n}}^{n}$, and as an easy observation we conclude that $x_{I J}(t)=0$ unless $i_{2}=j_{2}, \ldots, i_{n}=j_{n}$. Combining we have,

$$
\hat{\beta}_{t}\left(e_{12}^{1}\right)=\sum_{I \in \mathscr{I}^{\prime}} e_{12}^{1} e_{i_{2} i_{2}}^{2} \cdots e_{i_{n} i_{n}}^{n}\left(x_{I}(t)\right),
$$

$x_{I}(t) \in\left(\mathfrak{A}_{n}\right)^{c}, \mathscr{I}^{\prime}$ is the set of $(n-1)$-tuples $\left(i_{2}, \ldots, i_{n}\right)$, with all $i_{r}$ either 1 or 2 . Using [5] again, let $\phi_{n}, n \in \mathbf{N}$, be the conditional expectation of the trace $\tau$ from $\mathfrak{A}$ onto $\mathfrak{U}_{n}$. Then

$$
\phi_{n}\left(\hat{\beta}_{t}\left(e_{12}^{1}\right)\right)=\sum_{I \in \mathscr{I}^{\prime}} e_{12}^{1} e_{i_{2} i_{2}}^{2} \cdots e_{i_{n} i_{n}}^{n} \cdot c_{I}(t)
$$

where $c_{I}(t)=\tau\left(x_{I}(t)\right)$. Using the strong continuity of $\left\{\hat{\beta}_{t}\right\}$, it follows that for $0<\varepsilon<1$ and for a fixed interval $[-s, s] \subseteq \mathbf{R}$, there exists $n$ sufficiently large so that

$$
\left\|\phi_{n}\left(\hat{\beta}_{t}\left(e_{12}^{1}\right)\right)-\hat{\beta}_{t}\left(e_{12}^{1}\right)\right\|<\varepsilon / 2, \quad \text { all } t \in[-s, s] .
$$

But

$$
\left[\hat{\beta}_{t}\left(e_{12}^{1}\right)\right] * \hat{\beta}_{t}\left(e_{12}^{1}\right)=\hat{\beta}_{t}\left(e_{21}^{1} e_{12}^{1}\right)=\hat{\beta}_{t}\left(e_{22}^{1}\right)=e_{22}^{1}
$$

from Lemma 3.1(i), so

$$
\begin{aligned}
\varepsilon & >\left\|\left[\phi_{n}\left(\hat{\beta}_{t}\left(e_{12}^{1}\right)\right)\right] * \phi_{n}\left(\hat{\beta}_{t}\left(e_{12}^{1}\right)\right)-e_{22}^{1}\right\| \\
& =\left\|\sum_{I \in \mathscr{I}^{\prime}} e_{22}^{1} e_{i_{2} i_{2}}^{2} \cdots e_{i_{n} i_{n}}^{n}\left|c_{I}(t)\right|^{2}-e_{22}^{1}\right\| \\
& =\left\|\sum_{I \in \mathscr{I}^{\prime}}\left(\left|c_{I}(t)\right|^{2}-1\right) e_{22}^{1} e_{i_{2} i_{2}}^{2} \cdots e_{i_{n} i_{n}}^{n}\right\| \\
& =\max _{I \in \mathscr{I}^{\prime}}\left\{\left|\left(\left|c_{I}(t)\right|^{2}-1\right)\right|\right\} .
\end{aligned}
$$


Therefore,

$$
\left|\left(\left|c_{I}(t)\right|-1\right)\right|<\varepsilon, \quad \text { all } I \in \mathscr{I}^{\prime} \text {, all } t \in[-s, s] .
$$

This establishes the first two parts of the following lemma.

LeMma 3.2. Let $h \in \mathscr{S}_{00}$, and let $\delta_{h}$ generate the one-parameter group $\left\{\beta_{t}: t \in \mathbf{R}\right\}$ on $\mathfrak{U}^{0}$. Suppose $\left\{\beta_{t}\right\}$ extends to a one-parameter group $\left\{\hat{\beta}_{t}: t \in \mathbf{R}\right\}$ of automorphisms on $\mathfrak{A}$. Then for $\varepsilon>0$ and positive $s \in \mathbf{R}$ there exists $n \in N$ and constants $c_{I}(t)$ ( $I \in \mathscr{I}^{\prime}$ the set of $(n-1)$-tuples $\left.\left(i_{2}, \ldots, i_{n}\right)\right)$ such that:

$$
\begin{gathered}
|| c_{I}(t)|-1|<\varepsilon, \quad \text { all } t \in[-s, s], \text { all } I \in \mathscr{I}^{\prime} . \\
\left\|\hat{\beta}_{t}\left(e_{12}^{1}\right)-\sum_{I \in \mathscr{I}^{\prime}} e_{12}^{1} e_{i_{2} i_{2}}^{2} \cdots e_{i_{n} i_{n}}^{n} c_{I}(t)\right\|<\varepsilon / 2, \quad \text { all } t \in[-s, s] .
\end{gathered}
$$

(iii) For any $m \geqslant n, t \in[-s, s]$, and any projection $P$ of the form $P=e_{j_{2} j_{2}}^{2} \cdots$ $e_{j_{m+1} j_{m+1}}^{m+1}$, where $j_{r}=1$ or 2 ,

$$
\left\|\hat{\beta}_{t}\left(e_{12}^{1}\right) P-e_{12}^{1} P c_{J}(t)\right\|<\varepsilon / 2, \quad \text { where } J=\left(j_{2}, \ldots, j_{n}\right) \in \mathscr{I}^{\prime} .
$$

Proof. Only (iii) remains to be shown. Multiplying the expression in (ii) by $P$ we have

$$
\begin{aligned}
\frac{\varepsilon}{2} & >\left\|\hat{\beta}_{t}\left(e_{12}^{1}\right) P-\sum_{I \in \mathscr{I}^{\prime}} e_{12}^{1} e_{i_{2} i_{2}}^{2} \cdots e_{i_{n} i_{n}}^{n} c_{I}(t) P\right\| \\
& =\left\|\hat{\beta}_{t}\left(e_{12}^{1}\right) P-c_{J}(t) e_{12}^{1} P\right\| .
\end{aligned}
$$

This completes the argument.

Finally, we may combine our results to provide a necessary condition for a generator $\delta_{h}\left(h \in \mathscr{S}_{00}\right)$ on $\mathfrak{A}^{0}$ to extend to a generator on $\mathfrak{A}$.

Lemma 3.3. Let $h=\left(h_{k}\right) \in \mathscr{S}_{00}, \delta_{h}$ and $\beta_{t}, t \in \mathbf{R}$, be as in Lemma 3.1. Suppose $\delta_{h}$ has an extension to a generator $\hat{\delta}_{h}$ on $\mathfrak{A}$ (equivalently, $\left\{\beta_{t}: t \in \mathbf{R}\right\}$ extends to a group of automorphisms $\left\{\hat{\beta}_{t}\right\}$ of $\left.\mathfrak{A}\right)$. Then Condition $\mathrm{C}^{\prime}$ of $\$ 2$ must hold.

Proof. For a fixed positive integer $k>2$, choose $\varepsilon$ such that

$$
0<\varepsilon<\left(\left|\exp \left(i / 2^{k}\right)-1\right|\right) / 2
$$

and choose $n$ sufficiently large so that conditions (i)-(iii) of Lemma 3.2 hold with $s=1$. Choose any finite strictly increasing set $\left\{l_{j}: 1 \leqslant j \leqslant q\right\}$ of positive integers with $n<l_{1}$, and let $m$ be any integer greater than $l_{q}+1$. Finally, let $r$ be an integer, $0 \leqslant r<l_{1}$, and consider any projection $P$ of the form

$$
P=e_{i_{2} i_{2}}^{2} \cdots e_{i_{1} i_{1} l_{1}}^{l_{1}} e_{22}^{l_{1}+1} \cdots e_{22}^{m},
$$

with $r=\#\left\{k: i_{k}=1,2 \leqslant k \leqslant l_{1}\right\}$. Let $x \in \mathfrak{A}^{0}$ be the operator

$$
x=e_{21}^{l_{1}+1} e_{21}^{l_{2}+1} \cdots e_{21}^{l^{q}+1} \cdot e_{12}^{m+1} e_{12}^{m+2} \cdots e_{12}^{m+q}
$$

we observe that

$$
\begin{aligned}
\beta_{t}\left(x^{*}\right) \hat{\beta}_{t}\left(e_{12}^{1}\right) P \beta_{t}(x) & =\hat{\beta}_{t}\left(e_{12}^{1}\right) \beta_{t}\left(x^{*}\right) P \beta_{t}(x) \\
& =\hat{\beta}_{t}\left(e_{12}^{1}\right) \beta_{t}\left(x^{*} P x\right)=\hat{\beta}_{t}\left(e_{12}^{1}\right) x^{*} P x
\end{aligned}
$$


where $\beta_{t}\left(x^{*} P x\right)=x^{*} P x$ follows from Lemma 3.1(i). Since $\|x\|=1$, we have, combining (11) and Lemma 3.2(iii),

$$
\begin{aligned}
& \| \beta_{t}\left(x^{*}\right)\left[e_{12}^{1} P c_{I}(t)\right] \beta_{t}(x)-e_{12}^{1} x^{*} P x c_{I}(t) \| \\
& \leqslant\left\|\beta_{t}\left(x^{*}\right)\left[e_{12}^{1} P c_{I}(t)\right] \beta_{t}(x)-\beta_{t}\left(x^{*}\right)\left[\hat{\beta}_{t}\left(e_{12}^{1}\right) P\right] \beta_{t}(x)\right\| \\
&+\left\|\hat{\beta}_{t}\left(e_{12}^{1}\right) x^{*} P x-e_{12}^{1} x^{*} P x c_{I}(t)\right\| \\
& \leqslant\left\|e_{12}^{1} P c_{I}(t)-\hat{\beta}_{t}\left(e_{12}^{1}\right) P\right\|+\left\|\hat{\beta}_{t}\left(e_{12}^{1}\right) x^{*} P x-e_{12}^{1} x^{*} P x c_{I}(t)\right\| \\
&<\varepsilon / 2+\left\|\hat{\beta}_{t}\left(e_{12}^{1}\right) x^{*} P x-e_{12}^{1} x^{*} P x c_{I}(t)\right\| .
\end{aligned}
$$

Next note that $x^{*} P x$ is a projection satisfying $e_{I I} x^{*} P x=x^{*} P x$, where $e_{I I}=e_{i_{2} i_{2}}^{2}$ $\cdots e_{i_{n} i_{n}}^{n}$, so another application of Lemma 3.2(iii) yields

$$
\left\|\hat{\beta}_{t}\left(e_{12}^{1}\right) x^{*} P x-e_{12}^{1} x^{*} P x c_{I}(t)\right\| \leqslant\left\|\hat{\beta}_{t}\left(e_{12}^{1}\right) e_{I I}-e_{12}^{1} e_{I I} c_{I}(t)\right\|<\varepsilon / 2,
$$

and therefore

$$
\left\|\beta_{t}\left(x^{*}\right)\left[e_{12}^{1} P c_{I}(t)\right] \beta_{t}(x)-e_{12}^{1} x^{*} P x c_{I}(t)\right\|<\varepsilon, \quad \text { for } t \in[-1,1] .
$$

But clearly $\left|c_{I}(t)\right|>1 / 2$ by Lemma 3.2(i) and the choice of $\varepsilon$, so

$$
\left\|\beta_{t}\left(x^{*}\right) e_{12}^{1} P \beta_{t}(x)-e_{12}^{1} x^{*} P x\right\|<2 \varepsilon, \quad t \in[-1,1] .
$$

We now compute $\beta_{t}\left(x^{*}\right) e_{12}^{1} P \beta_{t}(x)$, beginning with

$$
\beta_{t}\left(e_{12}^{l_{1}+1} e_{21}^{m+1}\right) e_{12}^{1} P \beta_{t}\left(e_{21}^{l_{1}+1} e_{12}^{m+1}\right)
$$

By Lemma 3.1(ii),

$$
\begin{aligned}
\beta_{t}\left(e_{21}^{l_{1}+1} e_{12}^{m+1}\right)= & \exp \left(i t \sum_{j=l_{1}+1}^{m-1} \sum_{l=0}^{j-1} F_{l}^{l_{1}+1, j}\left(\xi_{l}^{j}-\xi_{l+1}^{j}\right) e_{11}^{j+1}\right) \\
& \cdot \exp \left(-i t \sum_{l=0}^{l_{1}-1} E_{l}^{l_{1}} \xi_{l}^{l_{1}}\right) \exp \left(i t \sum_{l=0}^{m-1} F_{l}^{l_{1}+1, m} \xi_{l}^{m}\right) e_{21}^{l_{1}+1} e_{12}^{m+1}
\end{aligned}
$$

Noting that

$$
\operatorname{Ad}\left[\exp \left(-i t \sum_{j=l_{1}+1}^{m-1} \sum_{l=0}^{j-1} F_{l}^{l_{1}+1, j}\left(\xi_{l}^{j}-\xi_{l+1}^{j}\right) e_{11}^{j+1}\right)\right]\left(e_{12}^{1} P\right)=e_{12}^{1} P
$$

our expression simplifies to

$$
\begin{aligned}
\beta_{t}\left(e_{12}^{l_{1}+1} e_{21}^{m+1}\right) e_{12}^{1} P \beta_{t}\left(e_{21}^{l_{1}+1} e_{12}^{m+1}\right) & \\
= & \left(e_{12}^{l_{1}+1} e_{21}^{m+1}\right) \exp \left(-i t \sum_{l=0}^{m-1} F_{l}^{l_{1}+1, m} \xi_{l}^{m}\right) \exp \left(i t \sum_{l=0}^{l_{1}-1} E_{l}^{l_{1}} \xi_{l}^{l_{1}}\right) e_{12}^{1} \\
& \cdot P \exp \left(-i t \sum_{l=0}^{l_{1}-1} E_{l}^{l_{1}} \xi_{l}^{l_{1}}\right) \exp \left(i t \sum_{l=0}^{m-1} F_{l}^{l_{1}+l, m} \xi_{l}^{m}\right) e_{21}^{l_{1}+1} e_{12}^{m+1}
\end{aligned}
$$


But recalling the expression for $P$, we have

$$
\begin{aligned}
\exp \left(i t \sum_{l=0}^{l_{1}-1} E_{l}^{l_{1}} \xi_{l}^{l_{1}}\right) & e_{12}^{1} e_{i_{2} i_{2}}^{2} \cdots e_{i_{1} i_{1}}^{l_{1}} e_{22}^{l_{1}+1} \cdots e_{22}^{m} \exp \left(-i t \sum_{l=0}^{l_{1}-1} E_{l}^{l_{1}} \xi_{l}^{l_{1}}\right) \\
& =\exp \left(i t \xi_{r+1}^{l_{1}}\right) e_{12}^{1} P \exp \left(-i t \xi_{r}^{l_{1}}\right)=\exp \left(i t\left[\xi_{r+1}^{l_{1}}-\xi_{r}^{l_{1}}\right]\right) \cdot e_{12}^{1} P .
\end{aligned}
$$

Continuing (and using similar calculations) we have

$$
\begin{aligned}
\beta_{t}\left(e_{12}^{l_{1}+1} e_{21}^{m+1}\right) & e_{12}^{1} P \beta_{t}\left(e_{21}^{l_{1}+1} e_{12}^{m+1}\right) \\
= & \exp \left(i t\left[\xi_{r+1}^{l_{1}}-\xi_{r}^{l_{1}}\right]\right) e_{12}^{l_{1}+1} e_{21}^{m+1} \exp \left(-i t \sum_{l=0}^{m-1} F_{l}^{l_{1}+1, m} \xi_{l}^{m}\right) e_{12}^{1} \\
& \cdot P \exp \left(i t \sum_{l=0}^{m-1} F_{l}^{l_{1}+1, m} \xi_{l}^{m}\right) e_{21}^{l_{1}+1} e_{12}^{m+1} \\
= & \exp \left(i t\left[\xi_{r+1}^{l_{1}}-\xi_{r}^{l_{1}}\right]\right) \exp \left(-i t\left[\xi_{r+1}^{m}-\xi_{r}^{m}\right]\right) e_{12}^{l_{1}+1} e_{21}^{m+1} e_{12}^{1} P e_{21}^{l_{1}+1} e_{12}^{m+1} \\
= & \exp \left(i t\left[\xi_{r+1}^{l_{1}}-\xi_{r}^{l_{1}}\right]\right) \exp \left(-i t\left[\xi_{r+1}^{m}-\xi_{r}^{m}\right]\right) \\
& \cdot e_{12}^{1} e_{i_{2} i_{2}}^{2} \cdots e_{i_{1} l_{1} l_{1}}^{l_{1}} e_{11}^{l_{1}+1} e_{22}^{l_{1}+2} \cdots e_{22}^{m} e_{22}^{m+1} .
\end{aligned}
$$

To complete the calculations we write $\beta_{t}\left(x^{*}\right) e_{12}^{1} P \beta_{t}(x)$ as

$$
\left[\prod_{j=1}^{q} \beta_{t}\left(e_{21}^{l_{j}+1} e_{12}^{m+j}\right)\right] * e_{12}^{1} P\left[\prod_{j=1}^{q} \beta_{t}\left(e_{21}^{l_{j}+1} e_{12}^{m+j}\right)\right],
$$

and using successive calculations similar to the one above, we arrive at

$$
\begin{aligned}
\beta_{t}\left(x^{*}\right) e_{12}^{1} P \beta_{t}(x)= & {\left[\prod_{j=1}^{q} \exp \left(i t\left[\xi_{r+j}^{l_{j}}-\xi_{r+j-1}^{l_{j}}\right]\right)\right] } \\
& \cdot\left[\prod_{j=1}^{q} \exp \left(-i t\left[\xi_{r+j}^{m+j-1}-\xi_{r+j-1}^{m+j-1}\right]\right)\right] e_{12}^{1} x^{*} P x .
\end{aligned}
$$

From (12) we obtain

$$
\left|\left[\prod_{j=1}^{q} \exp \left(i t\left[\xi_{r+j}^{l_{j}}-\xi_{r+j-1}^{l_{j}}\right]\right)\right]\left[\prod_{j=1}^{q} \exp \left(-i t\left[\xi_{r+j}^{m+j-1}-\xi_{r+j-1}^{m+j-1}\right]\right)\right]-1\right|<2 \varepsilon .
$$

Now (recalling the condition on $\varepsilon$ ),

(13) $\left|\exp \left(i / 2^{k}\right)-1\right|>2 \varepsilon$

$$
>\left|\exp \left(i t\left[\sum_{j=1}^{q}\left(\xi_{r+j}^{l_{j}}-\xi_{r+j-1}^{l_{j}}\right)-\left(\xi_{r+j}^{m+j-1}-\xi_{r+j-1}^{m+j-1}\right)\right]\right)-1\right|,
$$

all $t \in[-1,1]$. Using (13) a straightforward argument now shows that for any finite increasing set $\left\{l_{j}: 1 \leqslant j \leqslant q\right\}$ of integers with $l_{1}>n, 0 \leqslant r<l_{1}$, and for $m>l_{q}+1$,

$$
\left|\sum_{j=1}^{q}\left(\xi_{r+j}^{l_{j}}-\xi_{r+j-1}^{l_{j}}\right)-\left(\xi_{r+j}^{m+1-1}-\xi_{r+j-1}^{m+j-1}\right)\right|<\frac{1}{2^{k}} \text {. }
$$


But this last inequality implies that Condition $\mathrm{C}^{\prime}$ must hold (since $k$ may be chosen arbitrarily), and this completes the proof of the lemma.

4. Existence of nonextendable derivations to the CAR algebra. We may now combine the results of the previous section to obtain necessary conditions for $\delta_{h}$, $h \in \mathscr{S}_{0}$, to extend to a generator on $\mathfrak{A}$. Using some of the results in [3] on derivations commuting with compact abelian group actions, we may apply our results to show (Corollary 4.1) that there exist generators on $\mathfrak{A}^{0}$ admitting no extensions to closed $*$-derivations on $\mathfrak{A}$.

THEOREM 4.1. Let $h=\left(h_{k}\right) \in \mathscr{S}_{0}$ be a sequence of operators-i.e.,

$$
h_{1}=0, \quad h_{k+1}-h_{k}=\sum_{l=0}^{k} E_{l}^{k} \gamma_{l}^{k} e_{11}^{k+1}, \quad \gamma_{l}^{k} \in \mathbf{R}, \quad \gamma_{k-1}^{k}=\gamma_{k}^{k},
$$

$\delta_{k}$ the corresponding generator on the GICAR algebra $\mathfrak{A}^{0}$. For $k \in \mathbf{N}, 0 \leqslant l \leqslant k$, let $\xi_{l}^{k}=\gamma_{l}^{k}-\gamma_{k}^{k}$. Then $\delta_{h}$ admits an extension to a generator on the CAR algebra $\mathfrak{A}$ only if Condition $\mathrm{C}^{\prime}$ (on the numbers $\xi_{l}^{k}$ ) of $\S 2$ holds.

Proof. First suppose that $\delta_{h}$ admits a generator extension $\hat{\delta}_{h}$ on $\mathfrak{A}$. Set $h^{\prime}=\left(h_{n}^{\prime}\right)$ where

$$
h_{n}^{\prime}=h_{n}+\sum_{k=1}^{n} \lambda_{k} e_{11}^{k}, \quad \text { and } \quad \lambda_{k}=-\gamma_{k-1}^{k-1}, \quad k \in \mathbf{N} .
$$

Applying Lemma $2.4, \delta_{h^{\prime}}$, must also have a generator extension. Moreover,

$$
\begin{aligned}
h_{n+1}^{\prime}-h_{n}^{\prime} & =h_{n+1}-h_{n}-\gamma_{n}^{n} e_{11}^{n+1} \\
& =\sum_{l=0}^{n} E_{l}^{n}\left(\gamma_{l}^{n}-\gamma_{n}^{n}\right) e_{11}^{n+1}=\sum_{l=0}^{n} E_{l}^{n}\left(\xi_{l}^{n}\right) e_{11}^{n+1},
\end{aligned}
$$

so that $h^{\prime} \in \mathscr{S}_{00}$ (note $\xi_{n-1}^{n}=\xi_{n}^{n}=0$ ). Therefore (Lemma 3.3) Condition $\mathrm{C}^{\prime}$ holds.

COROLlaRY. Let $h=\left(h_{n}\right) \in \mathscr{S}_{00}$ be a sequence for which $\delta_{h}$ has no generator extensions to $\mathfrak{A}$. Then there are no densely-defined closed $*$-derivations on $\mathfrak{A}$ extending $\delta_{h}$.

Proof. Suppose there exists a closed $*$-derivation $\delta$ on $\mathfrak{A}$ extending $\delta_{k}$; i.e., $D(\delta)$ is a dense *-subalgebra of $\mathfrak{A}, D(\delta) \supseteq D\left(\delta_{h}\right)$, and $\delta_{\mid D\left(\delta_{h}\right)}=\delta_{\hbar}$. By Lemma 3.1(i), $\beta_{t}^{h}\left(e_{i i}^{n}\right)=e_{i i}^{n}(i=1,2, n \in N)$, so $e_{i i}^{n} \in D\left(\delta_{h}\right)(\subset D(\delta))$, and clearly $\delta\left(e_{i i}^{n}\right)=\delta_{h}\left(e_{i i}^{n}\right)$ $=0$. Since $\delta$ is closed it is immediate that $\mathscr{C} \subset D(\delta)$, where $\mathscr{C}$ is the maximal abelian subalgebra of $\mathscr{A}$ generated by the diagonal elements $e_{i i}^{n}$, and that $\delta_{\mid \mathscr{B}}=0$. In particular, $\delta\left[V_{n}(\theta)\right]=0$ (see $\S 2$ ). Hence if $x \in D(\delta)$,

$$
\operatorname{Ad}\left(V_{n}(\theta)\right)(x) \in D(\delta) \text { and } \delta\left[\operatorname{Ad}\left(V_{n}(\theta)\right)(x)\right]=\operatorname{Ad}\left(V_{n}(\theta)\right)(\delta x) .
$$

Recall from (1) that

$$
\alpha_{\theta}(x)=\lim _{n} \operatorname{Ad}\left(V_{n}(\theta)\right)(x)
$$


(respectively,

$$
\left.\alpha_{\theta}(\delta x)=\lim _{n} \operatorname{Ad}\left(V_{n}(\theta)\right)(\delta x)=\lim _{n} \delta\left[\operatorname{Ad}\left(V_{n}(\theta)\right)(x)\right]\right) .
$$

Thus $\alpha_{\theta}(x) \in D(\delta)$, by the closedness of $\delta$, and $\delta\left(\alpha_{\theta}(x)\right)=\alpha_{\theta}(\delta x)$. But $\delta$ also enjoys the property that its restriction $\delta_{h}$ to the invariant subalgebra $\mathfrak{A}^{0}$ is a generator, and therefore [3, Theorem 3.1] $\delta$ is itself a generator on $\mathfrak{A}$, contrary to hypothesis. This contradiction yields the result.

REMARK (Condition C and uniform convergence of $\left.\left(h_{n}^{\prime}\right) \in \mathscr{S}_{00}\right)$. Suppose $h^{\prime}=\left(h_{n}^{\prime}\right)$ is a sequence in $\mathscr{S}_{00}$ (as in Definition 2.1) such that $\left\{h_{n}^{\prime}: n \in \mathbf{N}\right\}$ is a uniformly convergent sequence of operators. Then it is straightforward to show that $\delta_{h}$ extends to a generator $\delta^{\prime}$ on $\mathfrak{A}$ with one-parameter group $\left\{\beta_{t}^{\prime}\right\}$ given by

$$
\beta_{t}^{\prime}=\lim _{n} \operatorname{Ad}\left(\exp \left(i t h_{n}^{\prime}\right)\right) \text {. }
$$

Thus, by Lemma 3.3, Condition $\mathrm{C}^{\prime}$ must hold. In fact, one sees that Condition $\mathrm{C}$ holds also. This may be shown directly as follows. Using Definition 2.1 (ii') we have (for $m>n$ )

$$
0=\lim _{m, n}\left\|h_{m+1}^{\prime}-h_{n}^{\prime}\right\|=\lim _{m, n}\left\|\sum_{k=n}^{m} \sum_{l=0}^{k-1} E_{l}^{k} \xi_{l}^{k} e_{11}^{k+1}\right\| .
$$

Arguing as in the proof of Lemma 2.5, one may show that

$$
\left\|\sum_{k=n}^{m} \sum_{l=0}^{k-1} E_{l}^{k} \xi_{l}^{k} e_{11}^{k+1}\right\|=\max _{I \in \mathscr{I}}\left\|e_{I I} \cdot \sum_{k=n}^{m} \sum_{l=0}^{k-1} E_{l}^{k} \xi_{l}^{k} e_{11}^{k+1}\right\|,
$$

where $\mathscr{I}$ is the set of $(m+1)$-tuples $\left(i_{1}, \ldots, i_{m+1}\right), i_{j}=1$ or $2, e_{I I}=e_{i_{1} i_{1}}^{1} \cdots e_{i_{m+1}^{m} i_{m+1}}^{1_{1}}$. Then, arguing as in the proof of Lemma 3.3,

$$
\left\|e_{I I} \cdot \sum_{k=n}^{m} \sum_{l=0}^{k-1} E_{l}^{k} \xi_{l}^{k} e_{11}^{k+1}\right\|=\left|\sum_{j=1}^{q} \xi_{r+j-1}^{l_{j}}\right|,
$$

where $r=\#\left\{j: i_{j}=1,1 \leqslant j \leqslant l_{1}\right\}$, and, with $n \leqslant l_{1}<\cdots<l_{q} \leqslant m,\left\{l_{j}: 1 \leqslant j \leqslant\right.$ $q\}$ is the set of indices $l$ such that $i_{l+1}=1$. But then combining this last result with (14) obviously implies Condition C.

On the other hand, we have been unable to determine whether Condition $\mathrm{C}$ implies uniform convergence of the sequence $\left(h_{n}^{\prime}\right) \in \mathscr{S}_{00}$ (or even whether Condition $\mathrm{C}$ is a necessary condition for the extendability of a derivation $\delta_{h}\left(h \in \mathscr{S}_{00}\right)$ to $\left.\mathfrak{A}\right)$.

\section{REFERENCES}

1. B. M. Baker, Free states of the gauge-invariant canonical anticommutation relations, Trans. Amer. Math. Soc. 237 (1978), 35-61.

2. O. Bratteli, Inductive limits of finite-dimensional $C^{*}$-algebras, Trans. Amer. Math. Soc. 171 (1972), 195-234.

3. O. Bratteli and P. E. T. Jørgensen, Derivations commuting with abelian gauge actions on lattice systems, Comm. Math. Phys. 87 (1982), 353-364.

4. Unbounded derivations tangential to compact groups of automorphisms, J. Funct. Anal. 48 (1982), 107-133.

5. G. A. Elliott, Derivations of matroid $C^{*}$-algebras, Invent. Math. 9 (1970), 253-269. 
6. F. Goodman and P. E. T. Jørgensen, Unbounded derivations commuting with compact group actions, Comm. Math. Phys. 82 (1981), 399-405.

7. F. Goodman and A. Wassermann, Unbounded derivations commuting with compact group actions. II, Univ. of Pennsylvania preprint, 1981.

8. A. Ikunishi, Derivations in $C^{*}$-algebras commuting with compact actions, Tokyo Inst. of Technology preprint, 1982.

9. A. Kishimoto and D. W. Robinson, On unbounded derivations commuting with a compact group of *-automorphisms, Univ. of New South Wales preprint, 1981.

10. R. T. Powers, UHF algebras and their applications to representations of the anti-commutation relations, Cargese Lectures in Physics 4 (1970), 137-168.

11. R. T. Powers and G. Price, Derivations vanishing on $S(\infty)$, Comm. Math. Phys. 84 (1982), 439-447.

12. M. Saito, Thesis, Univ. of Pennsylvania, 1979.

13. S. Sakai, On commutative normal *-derivations, Comm. Math. Phys. 43 (1975), 39-40.

14. G. Stamatopoulos, Thesis, Univ. of Pennsylvania, 1974.

Department of Mathematics, United States Naval ACademy, AnNapolis, Maryland 21402 